\title{
Activin Selectively Abolishes Hippocampal Long-Term Potentiation Induced by Weak Tetanic Stimulation In Vivo
}

\author{
Yuji Ikegaya ${ }^{1}$, Hiroshi Saito ${ }^{1}$, Kunio Torii ${ }^{2,3}$ and Nobuyoshi Nishiyama ${ }^{1, *}$ \\ ${ }^{2}$ Department of Chemical Pharmacology, Faculty of Pharmaceutical Sciences, The University of Tokyo, Tokyo 113, Japan \\ ${ }^{2}$ Central Research Laboratories, Ajinomoto Co., Inc., Kawasaki 210, Japan \\ ${ }^{3}$ Torii Nutrient-Stasis Project, ERATO, R\&D Corp. of Japan, Yokohama 221, Japan
}

Received June 12, $1997 \quad$ Accepted July 14, 1997

\begin{abstract}
Although modulation of hippocampal synaptic plasticity by neurotrophins or growth factors has recently become an extensively investigated subject, there are no reports arguing for the contribution of the transforming growth factor (TGF)- $\beta$ superfamily. In the present study, we examined the effect of activin, a member of the TGF- $\beta$ superfamily, on long-term potentiation (LTP) of the dentate gyrus in anesthetized rats. Activin significantly impaired the formation of LTP induced by tetanic stimulation (60 $\mathrm{Hz}$ for $0.27 \mathrm{sec})$, but not that by strong tetanic stimulation $(60 \mathrm{~Hz}$ for $0.5 \mathrm{sec})$. These results suggest that activin selectively blocks the induction of LTP evoked by threshold tetanic stimulation.
\end{abstract}

Keywords: Hippocampus, Long-term potentiation, Activin

Studies of activity-dependent synaptic plasticity including long-term potentiation (LTP) that may underlie learning and memory have long postulated the existence of extracellular signaling molecules that modulate and stabilize synaptic function (1). In recent years, neurotrophins and growth factors have emerged as attractive candidates for such signaling molecules $(2,3)$. There are, especially, many reports concerning facilitatory modulation of synaptic plasticity by ligands for receptors containing the tyrosine-kinase domain such as brain-derived neurotrophic factor and basic fibroblast growth factor.

Activin is a multifunctional protein belonging to the transforming growth factor- $\beta$ superfamily and its receptors are coupled to protein serine/threonine kinase (4). Funaba et al. (5) recently indicated that type Il activin receptors are highly expressed in the adult hippocampus. Additionally, several reports show that activin A mRNA is induced in the hippocampal dentate gyrus following synaptic activity and the induction of LTP $(6,7)$. However, it is not known whether activin has any influences on hippocampal synaptic transmission and synaptic plasticity. In the present study, therefore, we tested the effect of activin on the formation of hippocampal LTP.

Recording of evoked potential was made as described

* To whom correspondence should be addressed. in our previous papers (8). Briefly, male Wistar rats, 8to 9-weeks-old, were fixed in a stereotaxic frame under anesthesia with urethane and $\alpha$-chloralose. The medial perforant path was stimulated, and the evoked potential was extracellularly recorded from the granule cell layer of the dentate gyrus. Test stimulation was applied at intervals of $30 \mathrm{sec}$, and its stimulus intensity was set to a level that evoked a population spike of half the maximum amplitude. Activin, dissolved in phosphate-buffered saline containing $0.1 \%$ bovine serum albumin, was injected for $90 \mathrm{sec}$ through a stainless steel cylindrical cannula inserted into the cerebroventricle $(0.8-\mathrm{mm}$ posterior to the bregma, $1.5-\mathrm{mm}$ lateral to the midline, $4.5-\mathrm{mm}$ ventral to the dura). Twenty minutes after the drug injection, weak or strong tetanic stimulation $(60 \mathrm{~Hz}$ for $0.27 \mathrm{sec}$ or 0.5 sec, respectively) was applied to induce LTP. LTP was evaluated by measuring changes in the population spike amplitude that was measured as described in Fig. 1. Recombinant human activin $\mathrm{A}$ was a gift from Dr. Y. Eto (Ajinomoto Co., Inc.).

In the vehicle-injected group, the hippocampal population spike was potentiated following weak tetanic stimulation $(60 \mathrm{~Hz}$ for $0.27 \mathrm{sec})$, and LTP was generated in all tested cases. When activin (100 ng, i.c.v.) was injected, there was no change in basal hippocampal responses before weak tetanic stimulation, but LTP was not induced (Figs. 1 and 2A). This LTP-blocking effect of activin was 
immediately before tetanus
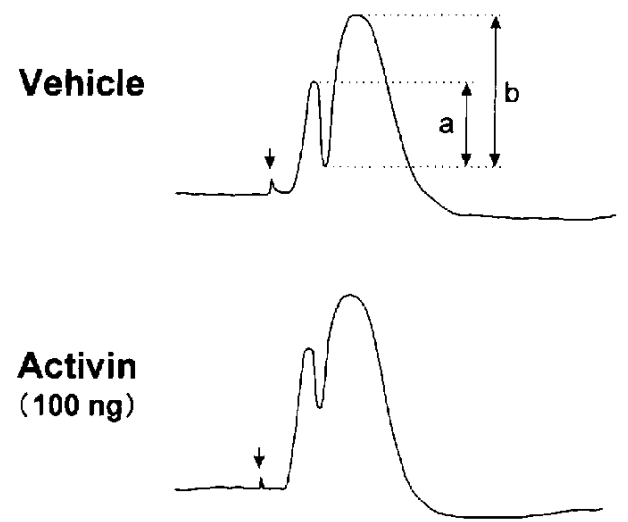

60 min after tetanus
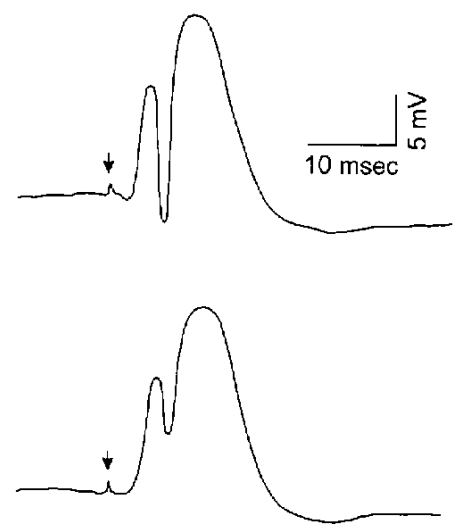

Fig. 1. Typical evoked potentials recorded from the dentate granule cell layer in the vehicle-injected group (upper traces) or the activin-injected group (down traces). Activin (100 ng, i.c.v.) was injected $20 \mathrm{~min}$ before tetanic stimulation. Left and right traces show field potentials immediately before and $60 \mathrm{~min}$ after tetanic stimulation, respectively. Test stimulation was delivered at the time indicated by arrows. The amplitude of the population spike was defined as the average of the amplitude from the first positive peak to the succeeding negative peak (a) and the amplitude from the negative peak to the second positive peak (b), i.e. $(\mathrm{a}+\mathrm{b}) / 2$
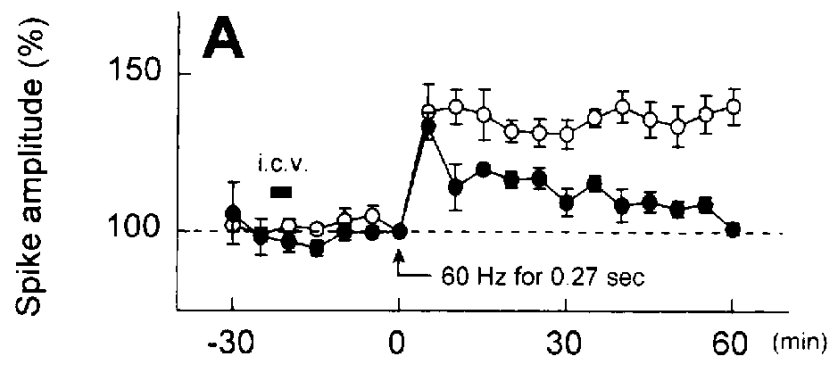

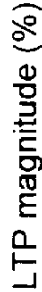

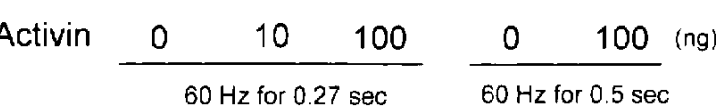

Fig. 2. Effect of activin on the induction of LTP in the dentate gyrus. A: time course of LTP induced by tetanic stimulation $(60 \mathrm{~Hz}$ for $0.27 \mathrm{sec}$ ) in the vehicle-injected group $(\bigcirc)$ and in the activininjected group (O). Activin (100 ng, i.c.v.) was injected during the time indicated by a black bar ( 22.5 to 20 min prior to tetanic stimulation), and tetanic stimulation was applied at time $0 \mathrm{~min}$. The population spike amplitude is expressed as a percentage of the baseline value immediately before tetanic stimulation. B: the dosedependent inhibitory effect of activin on the magnitude of LTP induced by a weak ( $60 \mathrm{~Hz}$ for $0.27 \mathrm{sec}$ ) or strong $(60 \mathrm{~Hz}$ for $0.5 \mathrm{sec})$ tetanic stimulation. The average of percent amplitude of population spikes 30 to 60 min after tetanic stimulation was calculated to compare the magnitude of LTP in each group. All reported data are means \pm S.E.M. $(n=5-6)$. An asterisk indicates a significant difference from the vehicle-injected group: ${ }^{*} \mathrm{P}<0.05$, Duncan's multiple range test following analysis of variance. dose-dependent (Fig. 2B). Finally we tested the effect of activin on LTP induced by strong tetanic stimulation ( 60 $\mathrm{Hz}$ for $0.5 \mathrm{sec}$ ). Activin at a dose of $100 \mathrm{ng}$ did not affect the induction of hippocampal LTP (Fig. 2B).

We have shown here that activin abolished the generation of hippocampal LTP induced by weak tetanic stimulation without affecting basal synaptic responses. There are many reports that ligands for receptor tyrosine kinase such as neurotrophins and growth factors modulate hippocampal synaptic plasticity $(2,3)$. However, this is the first report showing that receptor serine/threonine kinase may be involved in hippocampal LTP. Interestingly, the inhibitory influence of activin on LTP is in marked contrast to the facilitatory effects of ligands for receptor tyrosine kinase, suggesting a unique role of activin in hippocampal synaptic plasticity.

The final concentration of activin injected at the dose of $100 \mathrm{ng}$ in the cerebrospinal fluid is approximately estimated to be at the sub- $\mu \mathrm{g} / \mathrm{ml}$ level. This concentration is appropriate and sufficient to exert physiological effects of activin. Therefore, we concluded that activin has no effect on robust LTP induced by strong tetanic stimulation.

Although the mechanism through which activin inhibits LTP is not clear, we can make several speculations: 1) Receptors of activin are known to interact directly with FK506-binding protein-12, which forms a complex with FK506 and thereby inhibits activation of calcineurin (9). Calcineurin regulates the activity of $N$-methyl-D-aspartate (NMDA) receptors and its inhibitors block the induction of LTP (10). Importantly, calcineurin seems to play a significant role, in particular, in synaptic plasticity following weak tetanic stimulation (11), consistent with our 
result that activin selectively blocked LTP induced by weak tetanic stimulation. 2) $\alpha_{2}$-Macroglobulin, one of the major serum glycoproteins, has been identified as a soluble binding protein for activin (12). This molecule is synthesized by neurons and astrocytes in the brain, and its receptor is highly expressed in the hippocampus (13). It was recently reported that $\alpha_{2}$-macroglobulin inhibited the formation of hippocampals LTP (14). Therefore, it is possible that activin indirectly affects LTP via $\alpha_{2}$-macroglobulin. 3) Activin is known to elicit an acute increase in the intracellular concentration of $\mathrm{Ca}^{2+}\left(\left[\mathrm{Ca}^{2+}\right]_{\mathrm{i}}\right)$ in various cells. We also observed that activin increases $\left[\mathrm{Ca}^{2+}\right]_{i}$ in a subpopulation of cultured hippocampal neurons (Y. Iwahori et al., unpublished data). Although elevation of $\left[\mathrm{Ca}^{2+}\right]_{i}$ is required for the induction of LTP, untimely increase in $\left[\mathrm{Ca}^{2+}\right]_{i}$ rather causes inactivation of synaptic NMDA receptors and diminishes the ability of subsequent tetanic stimulation to induce LTP (15). In activintreated rats, therefore, preexistence of $\left[\mathrm{Ca}^{2+}\right]_{i}$ elevation may attenuate the generation of LTP.

Activin selectively blocked hippocampal LTP evoked by weak tetanic stimulation without affecting LTP by strong tetanic stimulation. These results can be interpreted as indicating that activin selectively extinguished threshold LTP and preserves robust LTP. Thus, activin may work as a permissive molecule to exclude ambiguous LTP and thereby to allow generation of only essential LTP.

\section{REFERENCES}

1 Bliss TVP and Collingridge GL: A synaptic model of memory: long-term potentiation in the hippocampus. Nature 361, 31-39 (1993)

2 Lo DG: Neurotrophic factors and synaptic plasticity. Neuron 15, 979-981 (1995)

3 Thoenen H: Neurotrophins and neuronal plasticity. Science 270, 593-598 (1995)
4 Massague J, Attisano L and Wrana JL: The TGF- $\beta$ family and its composite receptors. Trends Cell Biol 4, 172-178 (1994)

5 Funaba M, Murata T, Fujimura H, Murata $M$, Abe $M$ and Torii K: Immunolocalization of type $I$ or type II activin receptors in the rat brain. J Neuroendocrinol 9, 105-111 (1997)

6 Andreasson $\mathrm{K}$ and Worley PF: Induction of $\beta$-A activin expression by synaptic activity and during neocortical development, Neuroscience 69, 781 - 796 (1995)

7 Inokuchi K, Kato A, Hiraia K, Hishinuma $F$, Inoue $M$ and Ozawa $\mathrm{F}$ : Increase in activin $\beta \mathrm{A}$ mRNA in rat hippocampus during long-term potentiation. FEBS Lett 382, 48- 52 (1996)

8 Ikegaya $\mathrm{Y}$, Saito $\mathrm{H}$ and Matsuki N: Involvement of carbon monoxide in long-term potentiation in the dentate gyrus of anesthetized rats. Jpn J Pharmacol 64, 225 - 227 (1994)

9 Wang T, Donahoe PK and Zervos AS: Specific interaction of type I receptors of the TGF- $\beta$ family with the immunophilin FKBP-12. Science 265, 674-676 (1994)

10 Lu YF, Tomizawa K, Moriwaki A, Hayashi Y, Tokuda M, Itano T, Hatase $O$ and Matsui $\mathrm{H}$ : Calcineurin inhibitors, FK506 and cyclosporin $A$, suppress the NMDA receptor-mediated potentials and LTP, but not depotentiation in the rat hippocampus. Brain Res 729, 142-146 (1996)

11 Mulkey RM, Endo S, Shenolikar S and Malenka RC: Involvement of a calcineurin/inhibitor-1 phosphatase cascade in hippocampal long-term depression. Nature 369, 486-488 (1994)

12 Mather JP: Follistatins and $\alpha_{2}$-macroglobulin are soluble binding proteins for inhibin and activin. Horm Res 45, 207-210 (1996)

13 Ganter U, Strauss S, Jonas U, Weidemann A, Beyreuther K, Volk B, Berger M and Bauer J: Alpha-2-macroglobulin synthesis in interleukin-6-stimulated human neuronal (SH-SY5Y neuroblastoma) cells. Potential significance for the processing of Alzheimer beta-amyloid precursor protein. FEBS Lett 282, 127-131 (1991)

14 Cavus I, Koo PH and Teyler TJ: Inhibition of long-term potentiation development in rat hippocampal slice by $\alpha_{2}$ macroglobulin, an acute-phase protein in the brain. J Neurosci Res 43, 282-288 (1996)

15 Rosenmund C, Feltz A and Westbrook GL: Calcium-dependent inactivation of synaptic NMDA receptors in hippocampal neurons. J Neurophysiol 73, 427-430 (1995) 